\title{
ON THE BOUNDEDNESS OF MAXIMAL OPERATORS AND SINGULAR OPERATORS WITH KERNELS IN $L(\log L)^{\alpha}\left(\mathbf{S}^{n-1}\right)$
}

\author{
H. M. AL-QASSEM
}

Received 15 November 2005; Revised 20 May 2006; Accepted 28 May 2006

We establish the $L^{p}$-boundedness for a class of singular integral operators and a class of related maximal operators when their singular kernels are given by functions $\Omega$ in $L(\log L)^{\alpha}\left(S^{n-1}\right)$.

Copyright (C) 2006 H. M. Al-Qassem. This is an open access article distributed under the Creative Commons Attribution License, which permits unrestricted use, distribution, and reproduction in any medium, provided the original work is properly cited.

\section{Introduction}

Throughout this paper, we let $x^{\prime}$ denote $x /|x|$ for $x \in \mathbb{R}^{n} \backslash\{0\}$ and let $p^{\prime}$ denote the conjugate index of $p$; that is, $1 / p+1 / p^{\prime}=1$. Also, we let $\mathbb{R}^{n}, n \geq 2$, denote the $n$-dimensional Euclidean space and let $\mathbf{S}^{n-1}$ denote the unit sphere in $\mathbb{R}^{n}$ equipped with the normalized Lebesgue measure $d \sigma=d \sigma(\cdot)$.

Let $\Gamma_{\phi}=\left\{(y, \phi(|y|)): y \in \mathbb{R}^{n}\right\}$ be the surface of revolution generated by a suitable function $\phi:[0, \infty) \rightarrow \mathbb{R}$. Let $K_{\Omega, h}(y)$ be a Calderón-Zygmund-type kernel of the form

$$
K_{\Omega, h}(y)=h(|y|) \Omega\left(y^{\prime}\right)|y|^{-n},
$$

where $h:[0, \infty) \rightarrow \mathbb{C}$ is a measurable function, and $\Omega$ is an integrable function over $\mathbf{S}^{n-1}$, satisfying

$$
\int_{S^{n-1}} \Omega(u) d \sigma(u)=0 .
$$

Let $L(\log L)^{\alpha}\left(\mathbf{S}^{n-1}\right)$ (for $\alpha>0$ ) denote the space of all those measurable functions $\Omega$ on $\mathbf{S}^{n-1}$ which satisfy

$$
\|\Omega\|_{L(\log L)^{\alpha}\left(\mathbf{S}^{n-1}\right)}=\int_{\mathbf{S}^{n-1}}|\Omega(y)| \log ^{\alpha}(2+|\Omega(y)|) d \sigma(y)<\infty .
$$


For $\gamma>1$, define $\Delta_{\gamma}\left(\mathbb{R}_{+}\right)$to be the set of all measurable functions $h$ on $\mathbb{R}_{+}$satisfying the condition

$$
\sup _{R>0}\left(R^{-1} \int_{0}^{R}|h(t)|^{\gamma} d t\right)^{1 / \gamma}<\infty
$$

and define $\Delta_{\infty}\left(\mathbb{R}_{+}\right)=L^{\infty}\left(\mathbb{R}_{+}\right)$. Also, for $\gamma \geq 1$, define $\mathscr{H}_{\gamma}\left(\mathbb{R}_{+}\right)$to be the set of all measurable functions $h$ on $\mathbb{R}_{+}$satisfying the condition $\|h\|_{L^{\gamma}\left(\mathbb{R}_{+}, d r / r\right)}=\left(\int_{\mathbb{R}_{+}}|h(r)|^{\gamma} d r / r\right)^{1 / \gamma} \leq 1$ and define $\mathscr{H}_{\infty}\left(\mathbb{R}_{+}\right)=L^{\infty}\left(\mathbb{R}_{+}, d t / t\right)$.

We remark at this point that $\Delta_{\infty}\left(\mathbb{R}_{+}\right) \varsubsetneqq \Delta_{\gamma_{2}}\left(\mathbb{R}_{+}\right) \varsubsetneqq \Delta_{\gamma_{1}}\left(\mathbb{R}_{+}\right)$for $\gamma_{1}<\gamma_{2}, \mathscr{H}_{\infty}\left(\mathbb{R}_{+}\right)=$ $\Delta_{\infty}\left(\mathbb{R}_{+}\right)$, and $\mathscr{H}_{\gamma}\left(\mathbb{R}_{+}\right) \varsubsetneqq \Delta_{\gamma}\left(\mathbb{R}_{+}\right)$for $1<\gamma<\infty$.

The purpose of this paper is to study the $L^{p}$ mapping properties of singular integral operators $T_{\phi, \Omega, h}$ in $\mathbb{R}^{n}$ along the surface of revolution $\Gamma_{\phi}$ defined for $\left(x, x_{n+1}\right) \in \mathbb{R}^{n} \times \mathbb{R}=$ $\mathbb{R}^{n+1}$ by

$$
\left(T_{\phi, \Omega, h} f\right)\left(x, x_{n+1}\right)=\text { p.v. } \int_{\mathbb{R}^{n}} f\left(x-y, x_{n+1}-\phi(|y|)\right) K_{\Omega, h}(y) d y .
$$

Also, we are interested in studying the $L^{p}$-boundedness of the related maximal operator $\mathfrak{J}_{\phi, \Omega}^{(\gamma)}$ given by

$$
\mathfrak{J}_{\phi, \Omega}^{(\gamma)} f\left(x, x_{n+1}\right)=\sup _{h \in \mathscr{H}_{\gamma}\left(\mathbb{R}_{+}\right)}\left|T_{\phi, \Omega, h} f\left(x, x_{n+1}\right)\right| .
$$

Whenever $\phi(t) \equiv 0$ and $h \equiv 1$, then $T_{\phi, \Omega, h}$ essentially is the classical Calderón-Zygmund singular integral operator $T_{\Omega}$ given by

$$
T_{\Omega} f(x)=\text { p.v. } \int_{\mathbb{R}^{n}} f(x-y) \Omega\left(y^{\prime}\right)|y|^{-n} d y .
$$

If $\phi(t) \equiv 0$, we will denote $T_{\phi, \Omega, h}$ by $T_{\Omega, h}$ and $\mathfrak{J}_{\phi, \Omega}^{(\gamma)}$ by $\mathfrak{J}_{\Omega}^{(\gamma)}$.

The investigation of the $L^{p}$-boundedness problem of the operator $T_{\Omega}$ began with Calderón and Zygmund in their well-known papers $[7,8]$. The operator $T_{\phi, \Omega, h}$, whose singular kernel has the additional roughness in the radial direction due to the presence of $h$, was first studied by Fefferman [17] and subsequently by other several well-known authors. For a sampling of past studies, see $[2,4,9,13,15,20]$. We will content ourselves here with recalling only the following pertinent results.

In their celebrated paper [8], Calderón and Zygmund showed that the $L^{p}$-boundedness of $T_{\Omega}$ holds for $1<p<\infty$ if $\Omega \in L \log L\left(\mathbf{S}^{n-1}\right)$. Moreover, the condition $\Omega \in L \log L\left(\mathbf{S}^{n-1}\right)$ turns out to be the most desirable size condition for the $L^{p}$-boundedness of $T_{\Omega}$. This was made clear by Calderón and Zygmund where it was shown that $T_{\Omega}$ may fail to be bounded on $L^{p}$ for any $p$ if the condition $\Omega \in L \log L\left(\mathbf{S}^{n-1}\right)$ is replaced by any weaker metric condition $\Omega \in L^{\varphi}\left(\mathbf{S}^{n-1}\right)$ with a $\varphi$ satisfying $\varphi(t)=o(t \log t)$ as $t \rightarrow \infty$ (e.g., $\varphi(t)=$ $\left.L(\log L)^{1-\varepsilon}\left(\mathbf{S}^{n-1}\right), 0<\varepsilon<1\right)($ see $[8])$. 
Kim et al. [18] studied the $L^{p}$-boundedness of $T_{\phi, \Omega, h}$ as described in the following theorem.

Theorem 1.1. Let $T_{\phi, \Omega, h}$ be given as in (1.5) and $h \equiv 1$. Assume that $\Omega \in C^{\infty}\left(\mathbf{S}^{n-1}\right)$ and satisfies (1.2). Assume also that $\phi(\cdot)$ is in $C^{2}$ of $[0, \infty)$, convex, and increasing. Then $T_{\phi, \Omega, 1}$ is bounded on $L^{p}\left(\mathbb{R}^{n+1}\right)$ for $1<p<\infty$.

Even though the authors of [18] imposed the condition $\Omega \in C^{\infty}\left(S^{n-1}\right)$ in Theorem 1.1 , the arguments employed in [18] can be modified to show that the conclusion in Theorem 1.1 remains valid when one weakens the condition on $\Omega$ from $\Omega \in C^{\infty}\left(\mathbf{S}^{n-1}\right)$ to $\Omega \in L^{q}\left(\mathbf{S}^{n-1}\right)$ for some $q>1$.

An improvement and extension over the above result was obtained by Al-Salman and Pan in [4], where the condition $\Omega \in L^{q}\left(\mathbf{S}^{n-1}\right)$ is replaced by the weaker condition $\Omega \in$ $L \log L\left(\mathbf{S}^{n-1}\right)$. In fact, they proved the following.

Theorem 1.2. Let $\phi$ be a $C^{2}$, convex, and increasing function satisfying $\phi(0)=0$. If $\Omega \in$ $L \log L\left(\mathbf{S}^{n-1}\right)$ and $h \in \Delta_{\gamma}\left(\mathbb{R}_{+}\right)$for some $\gamma>1$, then the operator $T_{\phi, \Omega, h}$ in (1.2)-(1.5) is bounded on $L^{p}\left(\mathbb{R}^{n+1}\right)$ for $|1 / p-1 / 2|<\min \left\{1 / \gamma^{\prime}, 1 / 2\right\}$.

We remark that the range of $p$ given in Theorem 1.2 is the full range $(1, \infty)$ whenever $\gamma \geq 2$. However, this range of $p$ becomes a tiny open interval around 2 as $\gamma$ approaches 1 . For $L^{p}$-boundedness results on singular integrals for $p$ satisfying $|1 / p-1 / 2|<$ $\min \left\{1 / \gamma^{\prime}, 1 / 2\right\}$, we refer the readers to $[2-4,14,15]$, among others. So, an unsolved problem is whether the $L^{p}$-boundedness of $T_{\phi, \Omega, h}$ holds for $p$ outside this range.

The main focus of this paper is to have a solution to the above problem. In fact, we have made some progress in resolving this problem by imposing a more restrictive condition on $h$. However, the price we paid in having a more restricted condition on $h$ is compensated by the fact that we are able to prove our results under a much weaker condition on $\Omega$. More precisely we prove the following.

Theorem 1.3. Let $T_{\phi, \Omega, h}$ be given as in (1.2)-(1.5) and let $\phi$ be a $C^{2}$, convex, and increasing function satisfying $\phi(0)=0$. Suppose that $h \in \mathcal{H}_{\gamma}\left(\mathbb{R}_{+}\right)$for some $1<\gamma \leq \infty$ and $\Omega \in L(\log L)^{1 / \gamma^{\prime}}\left(\mathbf{S}^{n-1}\right)$. Then $T_{\phi, \Omega, h}$ is bounded on $L^{p}\left(\mathbb{R}^{n}\right)$ for $1<p<\infty$.

At this point, it is worth mentioning that the proof of Theorem 1.3 cannot be obtained by a simple application of existing arguments on singular integrals. Even though we have a more restrictive condition on $h$, if we try to apply previously known arguments, then we can prove our result only for $p$ satisfying $|1 / p-1 / 2|<\min \left\{1 / \gamma^{\prime}, 1 / 2\right\}$. To be able to obtain the $L^{p}$-boundedness for the full range $1<p<\infty$, a new maximal function that intervenes here in the proof of Theorem 1.3 is the maximal operator $\mathfrak{J}_{\phi, \Omega}^{(\gamma)}$ defined in (1.6). The study of the maximal operator $\mathfrak{J}_{\phi, \Omega}^{(\gamma)}$ began by Chen and Lin in [10] and subsequently by many other authors $[1,12,19]$. For example, Chen and Lin proved the following.

Theorem 1.4 [10]. Assume $n \geq 2,1 \leq \gamma \leq 2$, and $\Omega \in C\left(\mathbf{S}^{n-1}\right)$ satisfying (1.2). Then $\mathfrak{J}_{\Omega}^{(\gamma)}$ is bounded on $L^{p}\left(\mathbb{R}^{n}\right)$ for $(\gamma n)^{\prime}<p<\infty$. Moreover, the range of $p$ is the best possible.

Very recently, Al-Qassem improved the result in Theorem 1.4 as described in the following theorem. 
Theorem 1.5 [1]. Let $n \geq 2$ and let $\mathfrak{J}_{\Omega}^{(\gamma)}$ be given as in (1.6). Then

(a) if $\Omega \in L(\log L)^{1 / \gamma^{\prime}}\left(\mathbf{S}^{n-1}\right)$ and satisfies (1.2), then $\mathfrak{J}_{\Omega}^{(\gamma)}$ is bounded on $L^{p}\left(\mathbb{R}^{n}\right)$ for $\gamma^{\prime} \leq$ $p<\infty$;

(b) there exists an $\Omega$ which lies in $L(\log L)^{1 / 2-\varepsilon}\left(\mathbf{S}^{n-1}\right)$ for all $\varepsilon>0$ and satisfies (1.2) such that $\mathfrak{J}_{\Omega}^{(2)}$ is not bounded on $L^{2}\left(\mathbb{R}^{n}\right)$.

Our result regarding $\mathfrak{J}_{\phi, \Omega}^{(\gamma)}$ is the following.

THeOREM 1.6. Let $\mathfrak{J}_{\phi, \Omega}^{(\gamma)}$ be given as in (1.6) and let $\phi$ be a $C^{2}$, convex and increasing function satisfying $\phi(0)=0$. Suppose $\Omega \in L(\log L)^{1 / \gamma^{\prime}}\left(\mathbf{S}^{n-1}\right)$ and satisfies (1.2). Then $\mathfrak{J}_{\phi, \Omega}^{(\gamma)}$ is bounded on $L^{p}\left(\mathbb{R}^{n}\right)$ for $\gamma^{\prime} \leq p<\infty$ and $1<\gamma \leq 2$, and it is bounded on $L^{\infty}\left(\mathbb{R}^{n}\right)$ for $\gamma=1$.

Remarks 1.7. (1) In order to clarify the relations between Theorems 1.1, 1.2, 1.4, and 1.5 and theorems 1.3 and 1.6, we remark that on the unit sphere $S^{n-1}$, for any $q>1$, the following proper inclusions relations hold:

$$
\begin{gathered}
L^{q}\left(\mathbf{S}^{n-1}\right) \subset L(\log L)\left(\mathbf{S}^{n-1}\right) \subset H^{1}\left(\mathbf{S}^{n-1}\right) \subset L^{1}\left(\mathbf{S}^{n-1}\right), \\
L(\log L)^{\beta}\left(\mathbf{S}^{n-1}\right) \subset L(\log L)^{\alpha}\left(\mathbf{S}^{n-1}\right) \quad \text { if } 0<\alpha<\beta, \\
L(\log L)^{\alpha}\left(\mathbf{S}^{n-1}\right) \subset H^{1}\left(\mathbf{S}^{n-1}\right) \quad \forall \alpha \geq 1,
\end{gathered}
$$

while

$$
L(\log L)^{\alpha}\left(\mathbf{S}^{n-1}\right) \nsupseteq H^{1}\left(\mathbf{S}^{n-1}\right) \nsupseteq L(\log L)^{\alpha}\left(\mathbf{S}^{n-1}\right) \quad \forall 0<\alpha<1 .
$$

Here $H^{1}\left(\mathbf{S}^{n-1}\right)$ is the Hardy space on the unit sphere in the sense of Coifman and Weiss [11].

(2) For the case $h \in \mathscr{H}_{\infty}\left(\mathbb{R}_{+}\right)=L^{\infty}\left(\mathbb{R}_{+}\right)$, the authors in [5] showed that there is a function $f \in L^{p}$ such that the maximal operator acting on $f$ (i.e., $\mathfrak{I}_{\Omega}^{(\infty)}(f)$ ) yields an identically infinite function. It is still an open question whether the $L^{p}$-boundedness of $\mathfrak{J}_{\Omega}^{(\gamma)}$ holds for $2<\gamma<\infty$. A point worth noting is that Theorem 1.3 implies the $L^{p_{-}}$ boundedness of $T_{\phi, \Omega, h}$ if $h \in \mathscr{H}_{\gamma}\left(\mathbb{R}_{+}\right)$for all $1<\gamma \leq \infty$.

(3) We notice that the singular integral operators $T_{\Omega, h}$ are bounded on $L^{p}$ if $\Omega \in$ $L(\log L)^{1 / \gamma^{\prime}}\left(\mathbf{S}^{n-1}\right)$ and $h \in \mathscr{H}_{\gamma}\left(\mathbb{R}_{+}\right)$for some $\gamma>1$, while the classical Calderón-Zygmund singular integral operator $T_{\Omega}=T_{\Omega, 1}$ is bounded on $L^{p}$ if $\Omega \in L(\log L)\left(\mathbf{S}^{n-1}\right)$. The reason for this new phenomenon on singular integrals is that the singular operators $T_{\Omega, h}$ (with $h \in \mathcal{H}_{\gamma}\left(\mathbb{R}_{+}\right)$for some $\left.1<\gamma<\infty\right)$ have weaker singularities than the singular operators $T_{\Omega, 1}$ due to the presence of the strong condition on $h$.

(4) We notice that Theorem 1.6 represents an improvement and extension over the result in Theorem 1.4 and it is an extension over Theorem 1.5. Also, since $L \log L\left(\mathbf{S}^{n-1}\right) \subset$ $L(\log L)^{1 / \gamma^{\prime}}\left(\mathbf{S}^{n-1}\right)$ for any $\gamma>1$, Theorem 1.3 represents an improvement over Theorem 1.2 in the case $h \in \mathscr{H}_{\gamma}\left(\mathbb{R}_{+}\right)$for some $1<\gamma<\infty$.

(5) The method employed in this paper is based in part on a combination of ideas and arguments from $[2,13,15,16,19]$, among others.

Throughout the rest of the paper, the letter $C$ will stand for a constant but not necessarily the same one in each occurrence. 


\section{Some basic lemmas}

Let us begin this section with the following definition.

Definition 2.1. For an arbitrary function $\phi(\cdot)$ on $\mathbb{R}_{+}, a_{\mu}>1$, and $\Omega_{\mu}: S^{n-1} \rightarrow \mathbb{R}$ with $\mu \in$ $\mathbb{N} \cup\{0\}$, define the sequence of measures $\left\{\sigma_{\phi, k, \mu}: k \in \mathbf{Z}\right\}$ and the corresponding maximal operator $\sigma_{\phi, \mu}^{*}$ on $\mathbb{R}^{n+1}$ by

$$
\begin{gathered}
\int_{\mathbb{R}^{n+1}} f d \sigma_{\phi, k, \mu}=\int_{a_{\mu}^{k} \leq|u|<a_{\mu}^{k+1}} f(u, \phi(|u|)) K_{\Omega_{\mu}, h}(u) d u, \\
\sigma_{\phi, \mu}^{*}(f)=\sup _{k \in \mathbf{Z}}|| \sigma_{\phi, k, \mu}|* f| .
\end{gathered}
$$

Now let us establish the following Fourier transform estimates that will be used in later sections. One of the key points in these Fourier transform estimates is that the radial nature of the hypersurface $\Gamma_{\phi}(x)=(x, \phi(|x|))$ allows one to obtain these estimates without any condition on $\phi$.

Lemma 2.2. Let $\mu \in \mathbb{N} \cup\{0\}, a_{\mu}=2^{(\mu+1)}$, and let $\phi(\cdot)$ be an arbitrary function on $\mathbb{R}_{+}$. Let $\Omega_{\mu}(\cdot)$ be a function on $\mathbf{S}^{n-1}$ satisfying the following conditions: (i) $\left\|\Omega_{\mu}\right\|_{L^{2}\left(\mathbf{S}^{n-1}\right)} \leq a_{\mu}^{2}$, (ii) $\left\|\Omega_{\mu}\right\|_{L^{1}\left(\mathbf{S}^{n-1}\right)} \leq 1$, and (iii) $\Omega_{\mu}$ satisfies the cancellation conditions in (1.2) with $\Omega$ replaced by $\Omega_{\mu}$. Let

$$
I_{\mu, k}(\xi, \eta)=\left(\int_{a_{\mu}^{k}}^{a_{\mu}^{k+1}}\left|\int_{S^{n-1}} \Omega_{\mu}(x) e^{-i(t \xi \cdot x+\eta \phi(t))} d \sigma(x)\right|^{2} \frac{d t}{t}\right)^{1 / 2}
$$

Then there exist positive constants $C$ and $\alpha$ such that

$$
\begin{gathered}
\left|I_{\mu, k}(\xi, \eta)\right| \leq C(\mu+1)^{1 / 2}, \\
\left|I_{\mu, k}(\xi, \eta)\right| \leq C(\mu+1)^{1 / 2}\left|a_{\mu}^{k} \xi\right|^{ \pm \alpha /(\mu+1)},
\end{gathered}
$$

where $\xi \in \mathbb{R}^{n}, \eta \in \mathbb{R}$, and $t^{ \pm \alpha}=\inf \left\{t^{\alpha}, t^{-\alpha}\right\}$. The constants $C$ and $\alpha$ are independent of $k$, $\mu, \xi, \eta$ and $\phi(\cdot)$.

Proof. First, by condition (ii) on $\Omega_{\mu}$, it is easy to see that (2.3) holds. Next, by the cancellation properties of $\Omega_{\mu}$ and by a change of variable, we have

$$
\left|I_{\mu, k}(\xi, \eta)\right|^{2} \leq \int_{1}^{a_{\mu}}\left(\int_{S^{n-1}}\left|e^{-i\left\{a_{\mu}^{k} t \xi \cdot x+\eta \phi\left(a_{\mu}^{k} t\right)\right\}}-e^{-i \eta \phi\left(a_{\mu}^{k} t\right)}\right|\left|\Omega_{\mu}(x)\right| d \sigma(x)\right)^{2} \frac{d t}{t},
$$

which easily implies

$$
\left|I_{\mu, k}(\xi, \eta)\right| \leq C(\mu+1)^{1 / 2} a_{\mu}\left|a_{\mu}^{k} \xi\right|
$$

By combining both estimates in (2.3) and (2.6), we get

$$
\left|I_{\mu, k}(\xi, \eta)\right| \leq C(\mu+1)^{\beta / 2} a_{\mu}^{\beta}\left|a_{\mu}^{k} \xi\right|^{\beta}(\mu+1)^{(1-\beta) / 2}
$$


6 Boundedness of maximal and singular operators

for any $0<\beta<1$ and for some constant $C>0$. By the last estimate and by letting $\beta=$ $1 / 8(\mu+1)$, we get the estimate in (2.4) with $\alpha=1 / 8$ and with a plus sign in the exponent. To get the second estimate, we notice that

$$
\left|\int_{S^{n-1}} \Omega_{\mu}(x) e^{-i\left(t a_{\mu}^{k} \xi \cdot x+\eta \phi\left(t a_{\mu}^{k}\right)\right)} d \sigma(x)\right|^{2}=\int_{S^{n-1} \times \mathbf{S}^{n-1}} \Omega_{\mu}(x) \overline{\Omega_{\mu}(u)} e^{-i a_{\mu}^{k} t \xi \cdot(x-u)} d \sigma(x) d \sigma(u),
$$

which leads to

$$
\left|I_{\mu, k}(\xi, \eta)\right|^{2}=\int_{\mathbf{S}^{n-1} \times \mathbf{S}^{n-1}} \Omega_{\mu}(x) \overline{\Omega_{\mu}(u)}\left(\int_{1}^{a_{\mu}} e^{-i a_{\mu}^{k} t \xi \cdot(x-u)} \frac{d t}{t}\right) d \sigma(x) d \sigma(u) .
$$

By employing integration by parts, we get

$$
\left|\int_{1}^{a_{\mu}} e^{-i a_{\mu}^{k} t \xi \cdot(x-u)} \frac{d t}{t}\right| \leq C \min \left\{(\mu+1),(\mu+1)\left|a_{\mu}^{k} \xi \cdot(x-u)\right|^{-1}\right\}
$$

and hence

$$
\left|\int_{1}^{a_{\mu}} e^{-i a_{\mu}^{k} t \xi \cdot(x-u)} \frac{d t}{t}\right| \leq C\left((\mu+1)^{\beta}\left|a_{\mu}^{k} \xi\right|^{-\beta}\left|\xi^{\prime} \cdot(x-u)\right|^{-\beta}\right)(\mu+1)^{(1-\beta)} \quad \text { for any } 0<\beta<1 .
$$

By the last estimate and by letting $\beta=1 / 4$, we obtain

$$
\left|\int_{1}^{a_{\mu}} e^{-i a_{\mu}^{k} t \xi \cdot(x-u)} \frac{d t}{t}\right| \leq C(\mu+1)\left|a_{\mu}^{k} \xi\right|^{-1 / 4}\left|\xi^{\prime} \cdot(x-u)\right|^{-1 / 4}
$$

By Schwarz's inequality, condition (i) on $\Omega_{\mu}$, and (2.9)-(2.12), we get

$$
\left|I_{\mu, k}(\xi, \eta)\right|^{2} \leq C(\mu+1) a_{\mu}^{4}\left|a_{\mu}^{k} \xi\right|^{-1 / 4}\left(\int_{S^{n-1} \times \mathbf{S}^{n-1}}\left|\xi^{\prime} \cdot(x-u)\right|^{-1 / 2} d \sigma(x) d \sigma(u)\right)^{1 / 2} .
$$

Since the last integral is finite, we get

$$
\left|I_{\mu, k}(\xi, \eta)\right| \leq C(\mu+1)^{1 / 2} a_{\mu}^{2}\left|a_{\mu}^{k} \xi\right|^{-1 / 8}
$$

As above, by combining (2.14) with (2.3), we obtain the second estimate in (2.4). Lemma 2.2 is proved.

Lemma 2.3. Let $\mu \in \mathbb{N} \cup\{0\}, a_{\mu}=2^{(\mu+1)}, h \in \mathscr{H}_{\gamma}\left(\mathbb{R}_{+}\right)$for some $1<\gamma<\infty$, and let $\phi(\cdot)$ be an arbitrary function on $\mathbb{R}_{+}$. Let $\Omega_{\mu}(\cdot)$ be a function on $\mathbf{S}^{n-1}$ satisfying the following conditions: (i) $\left\|\Omega_{\mu}\right\|_{L^{2}\left(\mathbf{S}^{n-1}\right)} \leq a_{\mu}^{2}$, and (ii) $\left\|\Omega_{\mu}\right\|_{L^{1}\left(\mathbf{S}^{n-1}\right)} \leq 1$. Let

$$
R_{k, \mu}(\xi, \eta)=\int_{a_{\mu}^{k}}^{a_{\mu}^{k+1}}\left|\int_{S^{n-1}} \Omega_{\mu}(x) e^{-i(t \xi \cdot x+\eta \phi(t))} d \sigma(x)\right||h(t)| \frac{d t}{t} .
$$


Then there exist positive constants $C$ independent of $k, \phi$, and $\mu$ such that

$$
\begin{gathered}
\left|R_{k, \mu}(\xi, \eta)\right| \leq C(\mu+1)^{1 / \gamma^{\prime}} \\
\left|R_{k, \mu}(\xi, \eta)\right| \leq C(\mu+1)^{1 / \gamma^{\prime}}\left|a_{\mu}^{k} \xi\right|^{ \pm \alpha / \gamma^{\prime}(\mu+1)} .
\end{gathered}
$$

Proof. By Hölder's inequality, we have

$$
\begin{aligned}
& \left|R_{k, \mu}(\xi, \eta)\right| \\
& \quad \leq\left(\int_{a_{\mu}^{k}}^{a_{\mu}^{k+1}}|h(t)|^{\gamma} \frac{d t}{t}\right)^{1 / \gamma}\left(\int_{a_{\mu}^{k}}^{a_{\mu}^{k+1}}\left|\int_{S^{n-1}} \Omega_{\mu}(x) e^{-i(t \xi \cdot x+\eta \phi(t))} d \sigma(x)\right|^{\gamma^{\prime}} \frac{d t}{t}\right)^{1 / \gamma^{\prime}} \\
& \quad \leq\left(\int_{0}^{\infty}|h(t)|^{\gamma} \frac{d t}{t}\right)^{1 / \gamma}\left(\int_{a_{\mu}^{k}}^{a_{\mu}^{k+1}}\left|\int_{S^{n-1}} \Omega_{\mu}(x) e^{-i(t \xi \cdot x+\eta \phi(t))} d \sigma(x)\right|^{\gamma^{\prime}} \frac{d t}{t}\right)^{1 / \gamma^{\prime}} \\
& \quad \leq\left(\int_{a_{\mu}^{k}}^{a_{\mu}^{k+1}}\left|\int_{S^{n-1}} \Omega_{\mu}(x) e^{-i(t \xi \cdot x+\eta \phi(t))} d \sigma(x)\right|^{\gamma^{\prime}} \frac{d t}{t}\right)^{1 / \gamma^{\prime}} .
\end{aligned}
$$

Now, if $2 \leq \gamma^{\prime}<\infty$, by noticing that $\left|\int_{S^{n-1}} \Omega_{\mu}(x) e^{-i(t \xi \cdot x+\eta \phi(t))} d \sigma(x)\right| \leq 1$, we get

$$
\left|R_{k, \mu}(\xi, \eta)\right| \leq\left(\int_{a_{\mu}^{k}}^{a_{\mu}^{k+1}}\left|\int_{S^{n-1}} \Omega_{\mu}(x) e^{-i(t \xi \cdot x+\eta \phi(t))} d \sigma(x)\right|^{2} \frac{d t}{t}\right)^{1 / \gamma^{\prime}}
$$

and hence by Lemma 2.2 we easily get (2.17). On the other hand, if $1<\gamma^{\prime}<2$, (2.17) follows by Lemma 2.2 and Hölder's inequality. This finishes the proof of Lemma 2.3.

We will need the following lemma which has its roots in $[2,13,15]$. A proof of this lemma can be obtained by the same proof (with only minor modifications) as that of [2, Lemma 3.2]. We omit the details.

LEMmA 2.4. Let $\left\{\sigma_{k}: k \in \mathbf{Z}\right\}$ be a sequence of Borel measures on $\mathbb{R}^{n}$ and let $L: \mathbb{R}^{n} \rightarrow \mathbb{R}^{d}$ be a linear transformation. Suppose that for all $k \in \mathbf{Z}, \xi \in \mathbb{R}^{n}$, for some $a \in[2, \infty), \lambda>0, \alpha>0$, $C>0$, and for some $B>1$,

(i) $\left\|\sigma_{k}\right\| \leq C B^{\lambda}$;

(ii) $\left|\hat{\sigma}_{k}(\xi)\right| \leq C B^{\lambda}\left(a^{k B}|L(\xi)|\right)^{ \pm \alpha / B}$;

(iii) for some $p_{0} \in(2, \infty)$,

$$
\left\|\left(\sum_{k \in \mathbf{Z}}\left|\sigma_{k} * g_{k}\right|^{2}\right)^{1 / 2}\right\|_{p_{0}} \leq C B^{\lambda}\left\|\left(\sum_{k \in \mathbf{Z}}\left|g_{k}\right|^{2}\right)^{1 / 2}\right\|_{p_{0}}
$$

holds for arbitrary functions $\left\{g_{k}\right\}$ on $\mathbb{R}^{n}$. Then for $p_{0}^{\prime}<p<p_{0}$ there exists a positive constant $C_{p}$ such that

$$
\left\|\sum_{k \in \mathbf{Z}} \sigma_{k} * f\right\|_{p} \leq C_{p} B^{\lambda}\|f\|_{p}
$$


8 Boundedness of maximal and singular operators

holds for all $f$ in $L^{p}\left(\mathbb{R}^{n}\right)$. The constant $C_{p}$ is independent of $B$ and the linear transformation $L$.

Our aim now is to establish the following result.

Lemma 2.5. Let $h \in \mathscr{H}_{\gamma}\left(\mathbb{R}_{+}\right)$for some $\gamma>1$ and let $\Omega_{\mu}$ be a function satisfying conditions (i) and (ii) in Lemma 2.2. Assume $\phi$ is in $C^{2}([0, \infty))$, convex, and increasing. Then for $\gamma^{\prime}<$ $p \leq \infty$ and $f \in L^{p}\left(\mathbb{R}^{n+1}\right)$, there exists a positive constant $C_{p}$ which is independent of $\mu$ such that

$$
\left\|\sigma_{\phi, \mu}^{*}(f)\right\|_{p} \leq C_{p}(\mu+1)^{1 / \gamma^{\prime}}\|f\|_{p}
$$

Proof. Without loss of generality, we may assume that $\Omega_{\mu} \geq 0$ and $h \geq 0$. By Hölder's inequality, we have

$$
\sigma_{\phi, \mu}^{*}(f) \leq\left(\int_{a_{\mu}^{k}}^{a_{\mu}^{k+1}}|h(t)|^{\gamma} \frac{d t}{t}\right)^{1 / \gamma}\left(\Upsilon_{\mu}^{*}\left(|f| \gamma^{\gamma^{\prime}}\right)\right)^{1 / \gamma^{\prime}} \leq C\left(\Upsilon_{\mu}^{*}\left(|f|^{\gamma^{\prime}}\right)\right)^{1 / \gamma^{\prime}}
$$

where $\int_{\mathbb{R}^{n+1}} f d \Upsilon_{k, \mu}=\int_{a_{\mu}^{k}} \leq|u|<a_{\mu}^{k+1} f(u, \phi(|u|))|u|^{-n} \Omega_{\mu}\left(u^{\prime}\right) d u$ and $\Upsilon_{\mu}^{*}(f)=\sup _{k \in \mathbf{Z}}|| \Upsilon_{k, \mu} \mid *$ $f \mid$. Therefore, in order to prove (2.22), it suffices to prove that

$$
\left\|Y_{\mu}^{*}(f)\right\|_{L^{p}\left(\mathbb{R}^{n+1}\right)} \leq C_{p}(\mu+1)\|f\|_{L^{p}\left(\mathbb{R}^{n+1}\right)} \quad \text { for } 1<p \leq \infty .
$$

However, the proof of (2.24) follows by the same argument employed in the proof of [4, Lemma 4.7] and hence the proof of Lemma 2.5 is complete.

Lemma 2.6. Let $h \in \mathscr{H}_{\gamma}\left(\mathbb{R}_{+}\right)$for some $\gamma \geq 2$ and let $\Omega_{\mu}$ be a function on $\mathbf{S}^{n-1}$ satisfying conditions (i) and (ii) in Lemma 2.2. Let $\phi$ be in $C^{2}([0, \infty))$, convex, and an increasing function with $\phi(0)=0$. Then, for $\gamma^{\prime}<p<\infty$, there exists a positive constant $C_{p}$ which is independent of $\mu$ such that

$$
\left\|\left(\sum_{k \in \mathbf{Z}}\left|\sigma_{\phi, k, \mu} * g_{k}\right|^{2}\right)^{1 / 2}\right\|_{L^{p}\left(\mathbb{R}^{n+1}\right)} \leq C_{p}(\mu+1)^{1 / \gamma^{\prime}}\left\|\left(\sum_{k \in \mathbf{Z}}\left|g_{k}\right|^{2}\right)^{1 / 2}\right\|_{L^{p}\left(\mathbb{R}^{n+1}\right)}
$$

holds for arbitrary measurable functions $\left\{g_{k}\right\}$ on $\mathbb{R}^{n+1}$.

Proof. We follow a similar argument as in [6]. Let $\gamma^{\prime}<p<\infty$. By Hölder's inequality and the condition on $h$, we get

$$
\left|\sigma_{\phi, k, \mu} * g_{k}\left(x, x_{n+1}\right)\right|^{\gamma^{\prime}} \leq C \int_{a_{\mu}^{k}}^{a_{\mu}^{k+1}} \int_{S^{n-1}}\left|\Omega_{\mu}(y)\right|\left|g_{k}\left(x-y t, x_{n+1}-\phi(t)\right)\right|^{\gamma^{\prime}} d \sigma(y) \frac{d t}{t} .
$$


Let $d=p / \gamma^{\prime}$. For $\left\{g_{k}\right\} \in L^{d}\left(\mathbb{R}^{n+1}, l^{2}\right)$, by duality, there exists a nonnegative function $\omega \in$ $L^{d^{\prime}}\left(\mathbb{R}^{n+1}\right)$ such that $\|\omega\|_{L^{d^{\prime}}} \leq 1$ and

$$
\left\|\left(\sum_{k \in \mathbf{Z}}\left|\sigma_{\phi, k, \mu} * g_{k}\right|^{\gamma^{\prime}}\right)^{1 / \gamma^{\prime}}\right\|_{p}^{\gamma^{\prime}}=\int_{\mathbb{R}^{n+1}} \sum_{k \in \mathbf{Z}}\left|\sigma_{\phi, k, \mu} * g_{k}\left(x, x_{n+1}\right)\right|^{\gamma^{\prime}} \omega\left(x, x_{n+1}\right) d x d x_{n+1} .
$$

Therefore, by (2.27) and a change of variable, we get

$$
\left\|\left(\sum_{k \in \mathbf{Z}}\left|\sigma_{\phi, k, \mu} * g_{k}\right|^{\gamma^{\prime}}\right)^{1 / \gamma^{\prime}}\right\|_{p}^{\gamma^{\prime}} \leq C \int_{\mathbb{R}^{n+1}} \sum_{k \in \mathbf{Z}}\left|g_{k}\left(x, x_{n+1}\right)\right|^{\gamma^{\prime}} M_{\mu} \omega\left(x, x_{n+1}\right) d x d x_{n+1},
$$

where

$$
M_{\mu} \omega\left(x, x_{n+1}\right)=\sup _{k \in \mathbf{Z}} \int_{a_{\mu}^{k} \leq|y|<a_{\mu}^{k+1}} \omega\left(x+y, x_{n+1}+\phi(|y|)\right)\left|\Omega_{\mu}\left(y^{\prime}\right)\right||y|^{-n} d y .
$$

By Hölder's inequality, we obtain

$$
\left\|\left(\sum_{k \in \mathbf{Z}}\left|\sigma_{\phi, k, \mu} * g_{k}\right|^{\gamma^{\prime}}\right)^{1 / \gamma^{\prime}}\right\|_{p}^{\gamma^{\prime}} \leq C\left\|\left(\sum_{k \in \mathbf{Z}}\left|g_{k}\right|^{\gamma^{\prime}}\right)^{1 / \gamma^{\prime}}\right\|_{p}^{\gamma^{\prime}}\left\|M_{\mu} \omega\right\|_{d^{\prime}}
$$

By [4, Lemma 4.7], we have $\left\|M_{\mu} \omega\right\|_{L^{d^{\prime}}} \leq C_{p}(\mu+1)$ which in turn implies

$$
\left\|\left(\sum_{k \in \mathbf{Z}}\left|\sigma_{\phi, k, \mu} * g_{k}\right|^{\gamma^{\prime}}\right)^{1 / \gamma^{\prime}}\right\|_{p} \leq C(\mu+1)^{1 / \gamma^{\prime}}\left\|\left(\sum_{k \in \mathbf{Z}}\left|g_{k}\right|^{\gamma^{\prime}}\right)^{1 / \gamma^{\prime}}\right\|_{p} .
$$

Moreover, again by Lemma 2.5, we have

$$
\left\|\sup _{k \in \mathbf{Z}}\left|\sigma_{\phi, k, \mu} * g_{k}\right|\right\|_{p} \leq\left\|\sigma_{\phi, \mu}^{*}\left(\left(\sup _{k \in \mathbf{Z}}\left|g_{k}\right|\right)\right)\right\|_{p} \leq C_{p}(\mu+1)^{1 / \gamma^{\prime}}\left\|\left(\sup _{k \in \mathbf{Z}}\left|g_{k}\right|\right)\right\|_{p}
$$

By using the operator interpolation theorem between (2.31) and (2.32) and since $\gamma^{\prime} \in$ $[1,2]$, we get (2.25) which concludes the proof of the lemma.

We are now ready to present the proofs of our main results.

\section{Proofs of Theorems 1.3 and 1.6}

Since the proof of Theorem 1.3 will rely heavily on Theorem 1.6 as well as on its proof, we start by proving Theorem 1.6.

Proof of Theorem 1.6. Assume that $\Omega$ satisfies (1.2) and belongs to $L(\log L)^{1 / \gamma^{\prime}}\left(\mathbf{S}^{n-1}\right)$ and $1 \leq \gamma \leq 2$. For $\mu \in \mathbb{N}$, let $\mathbf{J}_{\mu}$ be the set of points $x \in \mathbf{S}^{n-1}$ which satisfy $2^{\mu} \leq|\Omega(x)|<2^{\mu+1}$. 
Also, we let $\mathbf{J}_{0}$ be the set of all those points $x \in \mathbf{S}^{n-1}$ which satisfy $|\Omega(x)|<2$. For $\mu \in$ $\mathbb{N} \cup\{0\}$, set $b_{\mu}=\Omega \chi_{\mathbf{J}_{\mu}}, \lambda_{0}=1$, and $\lambda_{\mu}=\left\|b_{\mu}\right\|_{1}$ for $\mu \in \mathbb{N}$. Set $I=\left\{\mu \in \mathbb{N}: \lambda_{\mu} \geq 2^{-2 \mu}\right\}$ and define the sequence of functions $\left\{\Omega_{\mu}\right\}_{\mu \in I \cup\{0\}}$ by

$$
\begin{gathered}
\Omega_{0}(x)=\sum_{\mu \in\{0\} \cup(\mathbb{N}-I)} b_{\mu}(x)-\sum_{\mu \in\{0\} \cup(\mathbb{N}-I)}\left(\int_{S^{n-1}} b_{\mu}(x) d \sigma(x)\right), \\
\Omega_{\mu}(x)=\left(\lambda_{\mu}\right)^{-1}\left(b_{\mu}(x)-\int_{S^{n-1}} b_{\mu}(x) d \sigma(x)\right) \quad \text { for } \mu \in I .
\end{gathered}
$$

For $\mu \in I \cup\{0\}$, set $a_{\mu}=2^{(\mu+1)}$. Then one can easily verify that the following hold for all $\mu \in I \cup\{0\}$ and for some positive constant $C$ :

$$
\begin{gathered}
\left\|\Omega_{\mu}\right\|_{2} \leq C a_{\mu}^{2}, \quad\left\|\Omega_{\mu}\right\|_{1} \leq C, \\
\sum_{\mu \in I \cup\{0\}}(\mu+1)^{1 / \gamma^{\prime}} \lambda_{\mu} \leq C\|\Omega\|_{L(\log L)^{1 / \gamma^{\prime}}\left(\mathbf{S}^{n-1}\right)}, \\
\int_{S^{n-1}} \Omega_{\mu}(u) d \sigma(u)=0 ; \quad \Omega=\sum_{\mu \in I \cup\{0\}} \lambda_{\mu} \Omega_{\mu} .
\end{gathered}
$$

By (3.4), we have $\mathfrak{J}_{\phi, \Omega}^{(\gamma)} f\left(x, x_{n+1}\right) \leq \sum_{\mu \in I \cup\{0\}} \lambda_{\mu} \mathfrak{J}_{\phi, \Omega_{\mu}}^{(\gamma)} f\left(x, x_{n+1}\right)$ and hence the proof of Theorem 1.6 is completed if we can show that

$$
\left\|\mathfrak{J}_{\phi, \Omega_{\mu}}^{(\gamma)} f\right\|_{L^{p}\left(\mathbb{R}^{n+1}\right)} \leq C_{p}(\mu+1)^{1 / \gamma^{\prime}}\|f\|_{L^{p}\left(\mathbb{R}^{n+1}\right)}
$$

holds for $\gamma^{\prime} \leq p<\infty$ if $1<\gamma \leq 2$ and for $p=\infty$ if $\gamma=1$. To prove (3.5), we need to consider three cases. We first prove (3.5) for the case $\gamma=2$.

Case $1(\gamma=2)$. By duality, we have

$$
\begin{aligned}
\mathfrak{J}_{\phi, \Omega_{\mu}}^{(2)} f\left(x, x_{n+1}\right) & =\sup _{h \in \mathcal{H}_{y}\left(\mathbb{R}_{+}\right)}\left|\int_{0}^{\infty} h(t) \int_{\mathbf{S}^{n-1}} f\left(x-t u, x_{n+1}-\phi(t)\right) \Omega_{\mu}(u) d \sigma(u) \frac{d t}{t}\right| \\
& =\left(\int_{0}^{\infty}\left|\int_{\mathbf{S}^{n-1}} f\left(x-t u, x_{n+1}-\phi(t)\right) \Omega_{\mu}(u) d \sigma(u)\right|^{2} \frac{d t}{t}\right)^{1 / 2} \\
& =\left(\sum_{k \in \mathbf{Z}} \int_{a_{\mu}^{k}}^{a_{\mu}^{k+1}}\left|\int_{\mathbf{S}^{n-1}} f\left(x-t u, x_{n+1}-\phi(t)\right) \Omega_{\mu}(u) d \sigma(u)\right|^{2} \frac{d t}{t}\right)^{1 / 2} .
\end{aligned}
$$

Let $\left\{\psi_{k, \mu}\right\}_{-\infty}^{\infty}$ be a smooth partition of unity in $(0, \infty)$ adapted to the interval $E_{k, \mu}=$ $\left[a_{\mu}^{-k-1}, a_{\mu}^{-k+1}\right]$. To be precise, we require the following: $\psi_{k, \mu} \in C^{\infty}, 0 \leq \psi_{k, \mu} \leq 1, \sum_{k} \psi_{k, \mu}(t)=$ 1 , supp $\psi_{k, \mu} \subseteq E_{k, \mu},\left|d^{s} \psi_{k, \mu}(t) / d t^{s}\right| \leq C_{s} / t^{s}$, where $C_{s}$ is independent of the lacunary sequence $\left\{a_{\mu}^{k}: k \in \mathbf{Z}\right\}$. Define the multiplier operators $S_{k, \mu}$ in $\mathbb{R}^{n+1}$ by

$$
\left(\widehat{S_{k, \mu} f}\right)(\xi, \eta)=\psi_{k, \mu}(|\xi|) \hat{f}(\xi, \eta) \quad \text { for }(\xi, \eta) \in \mathbb{R}^{n} \times \mathbb{R}
$$


Then for any $f \in \mathscr{S}\left(\mathbb{R}^{n+1}\right)$ and $l \in \mathbf{Z}$ we have $f\left(x, x_{n+1}\right)=\sum_{k \in \mathbf{Z}}\left(S_{k+l, \mu} f\right)\left(x, x_{n+1}\right)$. Thus, by (3.6) and applying Minkowski's inequality, we get

$$
\begin{aligned}
\mathfrak{J}_{\phi, \Omega_{\mu}}^{(2)} f\left(x, x_{n+1}\right) & \leq\left(\sum_{k \in \mathbf{Z}} \int_{a_{\mu}^{k}}^{a_{\mu}^{k+1}}\left|\sum_{l \in \mathbf{Z}} H_{k+l, t, \mu} f\left(x, x_{n+1}\right)\right|^{2} \frac{d t}{t}\right)^{1 / 2} \\
& \leq \sum_{l \in \mathbf{Z}}\left(\sum_{k \in \mathbf{Z}} \int_{a_{\mu}^{k}}^{a_{\mu}^{k+1}}\left|H_{k+l, t, \mu} f\left(x, x_{n+1}\right)\right|^{2} \frac{d t}{t}\right)^{1 / 2} \\
& =\sum_{l \in \mathbf{Z}} T_{l, \mu} f\left(x, x_{n+1}\right),
\end{aligned}
$$

where

$$
\begin{aligned}
H_{l, t, \mu} f\left(x, x_{n+1}\right) & =\int_{S^{n-1}}\left(S_{l, \mu} f\right)\left(x-t u, x_{n+1}-\phi(t)\right) \Omega_{\mu}(u) d \sigma(u), \\
T_{l, \mu} f\left(x, x_{n+1}\right) & =\left(\sum_{k \in \mathbf{Z}} \int_{a_{\mu}^{k}}^{a_{\mu}^{k+1}}\left|H_{k+l, t, \mu} f\left(x, x_{n+1}\right)\right|^{2} \frac{d t}{t}\right)^{1 / 2} .
\end{aligned}
$$

Therefore, to prove (3.5) for $\gamma=2$, it suffices to prove

$$
\left\|T_{l, \mu}(f)\right\|_{L^{p}\left(\mathbb{R}^{n+1}\right)} \leq C_{p}(\mu+1)^{1 / 2} 2^{-\theta_{p}|l|}\|f\|_{L^{p}\left(\mathbb{R}^{n+1}\right)}
$$

for some positive constants $C_{p}, \theta_{p}$, and for all $2 \leq p<\infty$.

The proof of (3.10) follows by interpolation between a sharp $L^{2}$ estimate and a cruder $L^{p}$ estimate of $T_{l, \mu}(f)$. First, the $L^{2}$-boundedness of $T_{l, \mu}(f)$ is provided by a simple application of Plancherel's theorem, Fubini's theorem and using Lemma 2.2:

$$
\begin{aligned}
\left\|T_{l, \mu}(f)\right\|_{2}^{2} & =\int_{\mathbb{R}} \int_{\mathbb{R}^{n}} \sum_{k \in \mathbf{Z}} \int_{a_{\mu}^{k}}^{a_{\mu}^{k+1}}\left|H_{k+l, t, \mu} f\left(x, x_{n+1}\right)\right|^{2} \frac{d t}{t} d x d x_{n+1} \\
& \leq \sum_{k \in \mathbf{Z}} \int_{\mathbb{R}} \int_{\Delta_{k+l}} \int_{a_{\mu}^{k}}^{a_{\mu}^{k+1}}\left|\Omega_{\mu}(x) e^{-i(t \xi \cdot x+\eta \phi(t))} d \sigma(x)\right|^{2} \frac{d t}{t}|\hat{f}(\xi, \eta)|^{2} d \xi d \eta \\
& \leq C(\mu+1) 2^{-2 \alpha|l|} \sum_{k \in \mathbf{Z}} \int_{\mathbb{R}} \int_{\Delta_{k+l}}|\hat{f}(\xi, \eta)|^{2} d \xi d \eta \\
& \leq C(\mu+1) 2^{-2 \alpha|l|}\|f\|_{2}^{2},
\end{aligned}
$$

where $\Delta_{k}=\left\{\xi \in \mathbb{R}^{n}:|\xi| \in E_{k, \mu}\right\}$. The last inequality holds since the sets $\Delta_{k}$ are finitely overlapping. Therefore, we have

$$
\left\|T_{l, \mu, \Omega_{\mu}}(f)\right\|_{2} \leq C(\mu+1)^{1 / 2} 2^{-\alpha|l|}\|f\|_{2} .
$$


12 Boundedness of maximal and singular operators

On the other hand, we need to compute the $L^{p}$-norm of $T_{l, \mu, \Omega_{\mu}}(f)$ for $p>2$. By duality, there is a function $g$ in $L^{(p / 2)^{\prime}}\left(\mathbb{R}^{n+1}\right)$ with $\|g\|_{(p / 2)^{\prime}} \leq 1$ such that

$$
\begin{aligned}
\left\|T_{l, \mu, \Omega_{\mu}}(f)\right\|_{p}^{2}= & \sum_{k \in \mathbf{Z}} \int_{\mathbb{R}^{n+1}} \int_{a_{\mu}^{k}}^{a_{\mu}^{k+1}}\left|H_{k+l, t, \Omega_{\mu}} f\left(x, x_{n+1}\right)\right|^{2} \frac{d t}{t}\left|g\left(x, x_{n+1}\right)\right| d x d x_{n+1} \\
\leq & \left\|\Omega_{\mu}\right\|_{1} \sum_{k \in \mathbf{Z}} \int_{\mathbb{R}^{n+1}} \int_{a_{\mu}^{k}}^{a_{\mu}^{k+1}} \int_{\mathbf{S}^{n-1}}\left|\Omega_{\mu}(u)\right|\left|g\left(x+t u, x_{n+1}+\phi(t)\right)\right| \\
& \times\left|S_{k+l, \mu} f\left(x, x_{n+1}\right)\right|^{2} d \sigma(u) \frac{d t}{t} d x d x_{n+1} \\
\leq & C \sum_{k \in \mathbf{Z}} \int_{\mathbf{R}^{n+1}}\left|S_{k+l, \mu} f\left(x, x_{n+1}\right)\right|^{2} \sigma_{\phi, \mu}^{*}(\tilde{g})\left(-x,-x_{n+1}\right) d x d x_{n+1} \\
\leq & C\left\|\sum_{k \in \mathbf{Z}}\left|S_{k+l, \mu} f\right|^{2}\right\|_{(p / 2)}\left\|\sigma_{\phi, \mu}^{*}(\tilde{g})\right\|_{(p / 2)^{\prime}},
\end{aligned}
$$

where $\tilde{g}\left(x, x_{n+1}\right)=g\left(-x,-x_{n+1}\right)$. By using Lemma 2.5, the Littlewood-Paley theory, and [21, Theorem 3] along with the remark that follows its statement in [21, page 96], we have

$$
\left\|T_{l, \mu, \Omega_{\mu}}(f)\right\|_{p} \leq C_{p}(\mu+1)^{1 / 2}\|f\|_{p} \quad \text { for } 2 \leq p<\infty .
$$

By interpolation between (3.12) and (3.14), we get (3.10) which ends the proof of (3.5) in the case $\gamma=2$.

Case $2(\gamma=1)$. If $f \in L^{\infty}\left(\mathbb{R}^{n+1}\right)$ and $h \in L^{1}\left(\mathbb{R}_{+}, d r / r\right)$, then

$$
\left|\int_{0}^{\infty} h(t) \int_{S^{n-1}} f\left(x-t u, x_{n+1}-\phi(t)\right) \Omega_{\mu}(u) d \sigma(u) \frac{d t}{t}\right| \leq C\|f\|_{L^{\infty}}\|h\|_{L^{1}\left(\mathbb{R}_{+}, d r / r\right)}
$$

for every $\left(x, x_{n+1}\right)$. Taking the supremum on both sides of the above inequality over all radial functions $h$ with $\|h\|_{L^{1}\left(\mathbb{R}_{+}, d r / r\right)} \leq 1$ yields

$$
\mathfrak{J}_{\phi, \Omega_{\mu}}^{(1)} f\left(x, x_{n+1}\right) \leq C\|f\|_{L^{\infty}\left(\mathbb{R}^{n+1}\right)}
$$

for almost every $\left(x, x_{n+1}\right) \in \mathbb{R}^{n+1}$. Hence,

$$
\left\|\mathfrak{J}_{\phi, \Omega_{\mu}}^{(1)} f\right\|_{L^{\infty}\left(\mathbb{R}^{n+1}\right)} \leq C\|f\|_{L^{\infty}\left(\mathbb{R}^{n+1}\right)}
$$

Case $3(1<\gamma<2)$. We will use an idea employed in [19]. By duality,

$$
\mathfrak{J}_{\phi, \Omega_{\mu}}^{(\gamma)} f\left(x, x_{n+1}\right)=\left\|\int_{S^{n-1}} f\left(x-t u, x_{n+1}-\phi(t)\right) \Omega_{\mu}(u) d \sigma(u)\right\|_{L^{\gamma^{\prime}}\left(\mathbb{R}_{+}, d t / t\right)} .
$$


Thus,

$$
\left\|\mathfrak{J}_{\phi, \Omega_{\mu}}^{(\gamma)} f\right\|_{L^{p}\left(\mathbb{R}^{n+1}\right)}=\|S(f)\|_{L^{p}\left(L^{y^{\prime}}\left(\mathbb{R}_{+}, d t / t\right), \mathbb{R}^{n+1}\right)},
$$

where $S: L^{p}\left(\mathbb{R}^{n+1}\right) \rightarrow L^{p}\left(L^{\gamma^{\prime}}\left(\mathbb{R}_{+}, d t / t\right), \mathbb{R}^{n+1}\right)$ defined by

$$
S(f)\left(x, x_{n+1}, t\right)=\int_{S^{n-1}} f\left(x-t u, x_{n+1}-\phi(t)\right) \Omega_{\mu}(u) d \sigma(u) .
$$

By (3.5) (for $\gamma=2$ ) and (3.17), we interpret that

$$
\|S(f)\|_{L^{p}\left(L^{2}\left(\mathbb{R}_{+}, d t / t\right), \mathbb{R}^{n+1}\right)} \leq C(\mu+1)^{1 / 2}\|f\|_{L^{p}\left(\mathbb{R}^{n+1}\right)}
$$

for $2<p<\infty$ and

$$
\|S(f)\|_{L^{\infty}\left(L^{\infty}\left(\mathbb{R}_{+}, d t / t\right), \mathbb{R}^{n+1}\right)} \leq C\|f\|_{L^{\infty}\left(\mathbb{R}^{n+1}\right)}
$$

Applying the real interpolation theorem for Lebesgue mixed normed spaces to the above results (see [6]), we conclude that

$$
\|S(f)\|_{L^{p}\left(L^{\gamma^{\prime}}\left(\mathbb{R}_{+}, d t / t\right), \mathbb{R}^{n+1}\right)} \leq C(\mu+1)^{1 / \gamma^{\prime}}\|f\|_{L^{p}\left(\mathbf{R}^{n+1}\right)}
$$

for $\gamma^{\prime} \leq p<\infty$ which in turn implies (3.5) for $1<\gamma<2$. The proof of Theorem 1.6 is complete.

Proof of Theorem 1.3. We divide the proof of Theorem 1.3 into three separate cases.

Case $1(1<\gamma \leq 2)$. As we pointed in the introduction, the proof of this case is based on Theorem 1.6. To this end, we argue as follows. Notice that

$$
\left(T_{\phi, \Omega, h} f\right)\left(x, x_{n+1}\right)=\lim _{\varepsilon \rightarrow 0} T_{\varepsilon} f\left(x, x_{n+1}\right),
$$

where $T_{\varepsilon}$ is the truncated singular integral operator given by

$$
T_{\varepsilon} f\left(x, x_{n+1}\right)=\int_{|y|>\varepsilon} f\left(x-y, x_{n+1}-\phi(|y|)\right) K_{\Omega, h}(y) d y .
$$

Without loss of generality, we may assume that $\|h\|_{L^{\gamma\left(\mathbb{R}_{+}, d r / r\right)}}=1$. Notice that, by Hölder's inequality, we have

$$
\begin{aligned}
\left|T_{\varepsilon} f\left(x, x_{n+1}\right)\right| & \leq \int_{\varepsilon}^{\infty}|h(t)|\left|\int_{S^{n-1}} f\left(x-t u, x_{n+1}-\phi(t)\right) \Omega(u) d \sigma(u)\right| \frac{d t}{t} \\
& \leq\left(\int_{0}^{\infty}\left|\int_{S^{n-1}} f\left(x-t u, x_{n+1}-\phi(t)\right) \Omega(u) d \sigma(u)\right|^{\gamma^{\prime}} \frac{d t}{t}\right)^{1 / \gamma^{\prime}} .
\end{aligned}
$$

Therefore,

$$
\left\|T_{\varepsilon} f\right\|_{p} \leq\left\|\mathfrak{J}_{\phi, \Omega}^{(\gamma)} f\right\|_{p} \leq C_{p}\|f\|_{p}
$$


for $\gamma^{\prime} \leq p<\infty$ and $1<\gamma \leq 2$, and $C$ is independent of $\varepsilon$. By a standard duality argument, $T_{\varepsilon}$ is bounded on $L^{p}\left(\mathbb{R}^{n+1}\right)$ for $1<p \leq \gamma$ and $1<\gamma \leq 2$. Passing to the limit as $\varepsilon \rightarrow 0$, Fatou's lemma gives $\left\|T_{\phi, \Omega, h} f\right\|_{p} \leq C\|f\|_{p}$ for $\gamma^{\prime} \leq p<\infty$ and for $1<p \leq \gamma$. If $\gamma=2$, then we are done; otherwise an application of the real interpolation theorem gives the $L^{p}$ boundedness of $T_{\phi, \Omega, h}$ for the remaining range of $p: \gamma<p<\gamma^{\prime}$.

Case $2(2<\gamma<\infty)$. As already seen in the proof of Theorem 1.6, by using the decomposition $\Omega=\sum_{\mu \in I \cup\{0\}} \lambda_{\mu} \Omega_{\mu}$, it suffices to show that

$$
\left\|T_{\phi, \Omega_{\mu}, h} f\right\|_{p} \leq C_{p}(1+\mu)^{1 / \gamma^{\prime}}\|f\|_{p} \quad \text { for } 1<p<\infty .
$$

To this end, we argue as above by considering $T_{\varepsilon}$ instead of $T_{\phi, \Omega, h}$. Write $T_{\varepsilon} f=\sum_{k \in Z} \sigma_{\phi, k, \mu} *$ $f$, one can then apply Lemmas $2.3,2.4$, and 2.6 to obtain

$$
\left\|T_{\varepsilon} f\right\|_{p} \leq C_{p}(1+\mu)^{1 / \gamma^{\prime}}\|f\|_{p}
$$

where $C_{p}$ is independent of $\varepsilon$. In particular,

$$
\left\|T_{\varepsilon} f\right\|_{p} \leq C_{p}(1+\mu)^{1 / \gamma^{\prime}}\|f\|_{p}
$$

for $2 \leq p<\infty$. By duality,

$$
\left\|T_{\varepsilon} f\right\|_{p} \leq C_{p}(1+\mu)^{1 / \gamma^{\prime}}\|f\|_{p}
$$

for $1<p \leq 2$. Thus, by Fatou's lemma, we have (3.28).

Case $3(\gamma=\infty)$. Finally, it follows directly from Theorem 1.2. This completes the proof of Theorem 1.3.

\section{Some additional results}

We will end the paper by presenting some additional results.

Theorem 4.1. Let $h \in \mathscr{H}_{\gamma}\left(\mathbb{R}_{+}\right)$for some $1<\gamma \leq \infty$. Let $\phi$ be a $C^{2}$, convex, and increasing function satisfying $\phi(0)=0$. Let $\Omega \in L(\log L)^{1 / \gamma^{\prime}}\left(\mathbf{S}^{n-1}\right)$ and satisfy (1.2). For each $\lambda \in \mathbb{R}$ define the oscillatory singular integral operator $\mathbf{S}_{\lambda}$ by

$$
\mathbf{S}_{\lambda} f(x)=\text { p.v. } \int_{\mathbb{R}^{n}} e^{i \lambda \phi(|x-y|)} \frac{\Omega(x-y)}{|x-y|^{n}} h(|x-y|) f(y) d y .
$$

Then the operators $\left\{\mathbf{S}_{\lambda}\right\}_{\lambda \in \mathbb{R}}$ are uniformly bounded on $L^{p}\left(\mathbb{R}^{n}\right)$ for $1<p<\infty$.

By a standard argument, Theorem 4.1 follows from Theorem 1.3. See the proof of [15, Theorem 9.1] for further details. 
Theorem 4.2. Let $h \in \mathscr{H}_{\gamma}\left(\mathbb{R}_{+}\right)$for some $1<\gamma \leq \infty$. Suppose that $\Omega \in L(\log L)^{1 / \gamma^{\prime}}\left(\mathbf{S}^{n-1}\right)$ and $\phi$ is a $C^{2}$, convex, and increasing function satisfying $\phi(0)=0$. Then the maximal operator $M_{\phi, \Omega, h}$ given by

$$
M_{\phi, \Omega, h} f\left(x, x_{n+1}\right)=\sup _{R>0}\left(R^{-n} \int_{|y|<R}\left|f\left(x-y, x_{n+1}-\phi(|y|)\right)\right||h(|y|)|\left|\Omega\left(y^{\prime}\right)\right| d y\right)
$$

is bounded on $L^{p}\left(\mathbb{R}^{n+1}\right)$ for $\gamma^{\prime}<p \leq \infty$.

Earlier the $L^{p}(1<p \leq \infty)$ boundedness of $\mathcal{M}_{\phi, \Omega, 1}$ was proved in [18] under the stronger condition that $\Omega \in L^{q}\left(\mathbf{S}^{n-1}\right)$ for some $q>1$ and later Al-Salman and Pan in [4] proved $L^{p}\left(\gamma^{\prime}<p \leq \infty\right)$ boundedness of $M_{\phi, \Omega, h}$ under the conditions $\Omega \in L \log L\left(\mathbf{S}^{n-1}\right)$ and $h \in$ $\Delta_{\gamma}\left(\mathbb{R}_{+}\right)$for some $\gamma>1$.

Proof of Theorem 4.2. By (3.4) we have

$$
M_{\phi, \Omega, h} f\left(x, x_{n+1}\right) \leq \sum_{\mu \in I \cup\{0\}} \lambda_{\mu} \mathcal{M}_{\phi,\left|\Omega_{\mu}\right|, h}
$$

and hence Theorem 4.2 follows by noticing that

$$
M_{\phi,\left|\Omega_{\mu}\right|, h}(f) \leq C \sigma_{\phi, \mu}^{*}(|f|)
$$

and invoking Lemma 2.5.

As usual, the pointwise existence of $T_{\phi, \Omega, h} f$ for $f$ in $L^{p}$ spaces can be established by considering the following maximal truncated singular integral

$$
T_{\phi, \Omega, h} f\left(x, x_{n+1}\right)=\sup _{\varepsilon>0}\left|T_{\varepsilon} f\left(x, x_{n+1}\right)\right|,
$$

where $T_{\varepsilon} f\left(x, x_{n+1}\right)$ is defined as in (3.25).

Theorem 4.3. Let $T_{\phi, \Omega, h}^{*}$ be given as above. Suppose that $h \in \mathscr{H}_{\gamma}\left(\mathbb{R}_{+}\right)$for some $1<\gamma \leq \infty$ and $\Omega \in L(\log L)^{1 / \gamma^{\prime}}\left(\mathbf{S}^{n-1}\right)$. Then $T_{\phi, \Omega, h}^{*}$ is bounded on $L^{p}\left(\mathbb{R}^{n+1}\right)$ for $\gamma^{\prime}<p<\infty$.

A proof of Theorem 4.3 can be constructed by using the above estimates and the arguments in [2] (see also [13, 15]). We omit the details.

\section{Acknowledgment}

The author is indebted to Professor Yibiao Pan for a helpful discussion.

\section{References}

[1] H. M. Al-Qassem, $L^{p}$ bounds for a class of rough maximal operators, preprint.

[2] H. M. Al-Qassem and Y. Pan, $L^{p}$ estimates for singular integrals with kernels belonging to certain block spaces, Revista Matemática Iberoamericana 18 (2002), no. 3, 701-730.

[3] Singular integrals along surfaces of revolution with rough kernels, SUT Journal of Mathematics 39 (2003), no. 1, 55-70. 
[4] A. Al-Salman and Y. Pan, Singular integrals with rough kernels in $\operatorname{Llog} L\left(\mathbf{S}^{n-1}\right)$, Journal of the London Mathematical Society. Second Series 66 (2002), no. 1, 153-174.

[5] J. M. Ash, P. F. Ash, C. L. Fefferman, and R. L. Jones, Singular integral operators with complex homogeneity, Studia Mathematica 65 (1979), no. 1, 31-50.

[6] A. Benedek and R. Panzone, The space $L^{p}$, with mixed norm, Duke Mathematical Journal 28 (1961), no. 3, 301-324.

[7] A. P. Calderón and A. Zygmund, On the existence of certain singular integrals, Acta Mathematica 88 (1952), no. 1, 85-139.

[8] _ On singular integrals, American Journal of Mathematics 78 (1956), 289-309.

[9] L.-K. Chen, On a singular integral, Studia Mathematica 85 (1986), no. 1, 61-72, 1987.

[10] L.-K. Chen and H. Lin, A maximal operator related to a class of singular integrals, Illinois Journal of Mathematics 34 (1990), no. 1, 120-126.

[11] R. R. Coifman and G. Weiss, Extensions of Hardy spaces and their use in analysis, Bulletin of the American Mathematical Society 83 (1977), no. 4, 569-645.

[12] Y. Ding and Q. He, Weighted boundedness of a rough maximal operator, Acta Mathematica Scientia. English Edition 20 (2000), no. 3, 417-422.

[13] J. Duoandikoetxea and J. L. Rubio de Francia, Maximal and singular integral operators via Fourier transform estimates, Inventiones Mathematicae 84 (1986), no. 3, 541-561.

[14] D. Fan and Y. Pan, A singular integral operator with rough kernel, Proceedings of the American Mathematical Society 125 (1997), no. 12, 3695-3703.

[15] _ Singular integral operators with rough kernels supported by subvarieties, American Journal of Mathematics 119 (1997), no. 4, 799-839.

[16] D. Fan, Y. Pan, and D. Yang, A weighted norm inequality for rough singular integrals, The Tohoku Mathematical Journal 51 (1999), no. 2, 141-161.

[17] R. Fefferman, A note on singular integrals, Proceedings of the American Mathematical Society 74 (1979), no. 2, 266-270.

[18] W.-J. Kim, S. Wainger, J. Wright, and S. Ziesler, Singular integrals and maximal functions associated to surfaces of revolution, The Bulletin of the London Mathematical Society 28 (1996), no. 3 , 291-296.

[19] H. V. Le, Maximal operators and singular integral operators along submanifolds, Journal of Mathematical Analysis and Applications 296 (2004), no. 1, 44-64.

[20] J. Namazi, A singular integral, Proceedings of the American Mathematical Society 96 (1986), no. 3, 421-424.

[21] E. M. Stein, Singular Integrals and Differentiability Properties of Functions, Princeton Mathematical Series, no. 30, Princeton University Press, New Jersey, 1970.

H. M. Al-Qassem: Department of Mathematics, Yarmouk University, Irbid, Jordan E-mail address: husseink@yu.edu.jo 